\title{
Quantitative EMCD by use of a double aperture for simultaneous acquisition of EELS
}

\author{
Hasan Ali ${ }^{\mathrm{a}}$, Tobias Warnatz ${ }^{\mathrm{b}}$, Ling Xie ${ }^{\mathrm{a}}$, Björgvin Hjörvarsson ${ }^{\mathrm{b}}$, Klaus Leifer ${ }^{\mathrm{a}}$ \\ ${ }^{a}$ Electron Microscopy and Nano-Engineering, Applied Materials Science, Department of Engineering Sciences, \\ Uppsala University, Box 534, 75121 Uppsala, Sweden \\ ${ }^{\mathrm{b}}$ Department of Physics and Astronomy, Uppsala University, Box 516, 75120 Uppsala, Sweden
}

Keywords: TEM; quantitative EMCD; double aperture; simultaneous acquisition; S/N ratio; magnetic moments

\begin{abstract}
:
The weak signal strength in electron magnetic circular dichroism (EMCD) measurements remains one of the main challenges in the quantification of EMCD related EELS spectra. As a consequence, small variations in peak intensity caused by changes of background intervals, choice of method for extraction of signal intensity and equally differences in sample quality can cause strong changes in the EMCD signal. When aiming for high resolution quantitative EMCD, an additional difficulty consists in the fact that the two angular resolved EELS spectra needed to obtain the EMCD signal are taken at two different instances and it cannot be guaranteed that the acquisition conditions for these two spectra are identical. Here, we present an experimental setup where we use a double hole aperture in the transmission electron microscope to obtain the EMCD signal in a single acquisition. This geometry allows for the parallel acquisition of the two electron energy loss spectra (EELS) under exactly the same conditions. We also compare the double aperture acquisition mode with the qE acquisition mode which has been previously used for parallel acquisition of EMCD. We show that the double aperture mode not only offers better signal to noise ratio as compared to $\mathrm{qE}$ mode but also allows for much higher acquisition times to significantly improve the signal quality which is crucial for quantitative analysis of the magnetic moments.
\end{abstract}

\section{Introduction:}

Electron magnetic circular dichroism (EMCD) is an electron energy loss spectroscopy (EELS) based technique to determine the magnetic moments of materials in the transmission electron microscope (TEM). The technique was introduced by Schattschneider and Hebert [1][2]. The EMCD technique is a counterpart of the well-established synchrotron based technique XMCD [3][4]. The major advantage of EMCD over XMCD is the promise to get the magnetic measurements with much higher spatial resolution, in principle with the resolution of the TEM, without the need of an expensive synchrotron source. The down side of the TEM and also, to some extent of the XMCD measurements are the very weak signal strength. During the last decade, various experimental acquisition geometries were proposed and shown to obtain an EMCD signal such as energy filtered diffraction patterns [5], energy filtered images [6][7][8] and using convergent electron beams [9][10]. A major effort has been to demonstrate magnetic information from EMCD experiments down to single atomic plane 
resolution [11][12]. Despite all these efforts and owed to the small magnetic signal in the EELS spectra the signal to noise $(\mathrm{S} / \mathrm{N})$ ratio is still a challenge in EMCD. Related to this, though of a different nature, it remains a challenge to obtain a reliable EMCD signal which can be used for the quantitative determination of the magnetic orbital to spin ratio $\mathrm{m}_{1} / \mathrm{m}_{\mathrm{s}}$. Whereas in the XMCD measurements, the diameter of the X-ray probe is typically between $50 \mathrm{~nm}$ to a few microns, in the TEM we aim at electron probe sizes with a diameter of only a few angstroms in the EMCD measurements. As well, the EMCD signal changes very sensitively with the sample orientation, a factor which plays minor or no role in XMCD experiments. A small change in the orientation or positioning of the electron probe can cause important changes in the resulting EMCD signal. Thus, in a TEM based measurement of magnetic dichroism, both sample position and sample orientation must be well known with an accuracy of 1 Angstrom and sub-0.1 mrad, respectively.

In the classical EMCD acquisition geometry that was introduced in 2003, the sample is tilted to a specific diffraction geometry called 2 beam condition (2BC) (or 3 beam condition) and two EELS spectra are acquired by placing the detector close to a Thales circle positions [13], the so called chiral plus $(\mathrm{C}+)$ and chiral minus (C-) positions. Most commonly, the two EELS spectra are acquired in a serial fashion by first placing an aperture at $\mathrm{C}+$ position and then moving it to $\mathrm{C}$ - position followed by an EELS acquisition at each position (Fig. 1a). Any change in experimental conditions between the two acquisitions would directly or indirectly influence the resulting EMCD signal and hence the quantitative measurements. For example, a small change in the orientation of the sample can significantly influence the resulting EMCD signal [7]. The specimen damage under the electron beam and the sample drift can also be the important factors to consider [9]. To avoid these points and with the prospect to obtain a reliable EMCD signal, one strategy consists in a simultaneous acquisition of the $\mathrm{C}+$ and C- EELS spectra, thus guaranteeing that they were acquired from the same atoms and on identical orientation. One such experimental set up has already been presented where both of the chiral EELS spectra can be acquired in parallel [14], the so called qE acquisition geometry. In this setup, the sample is rotated such that, at the two beam condition (2BC), the reciprocal space vector $\mathbf{G}$ is aligned parallel to the $\mathrm{q}_{\mathrm{x}}$ axis (zero beam - $\mathbf{G}$ axis) in the energy filtered diffraction pattern as shown in Fig. 1b. Switching to the spectroscopy mode under these conditions would replace the $q_{x}$ axis to the energy dispersion axis whereas the $\mathrm{q}_{\mathrm{y}}$ values are preserved and the resulting image is called the $\mathrm{qE}$ diagram or the qE EELS image (Fig. 1b). The chiral EELS spectra can be extracted from the qE image by line profiles parallel to $\mathrm{q}_{\mathrm{x}}$ and intersecting the $\mathrm{C}+$ and $\mathrm{C}-$ positions. As indicated in Fig. 1b, the resulting EELS spectrum contains data averaged along the $\mathrm{q}_{\mathrm{x}}$ direction, which means that most of the signal is collected at scatter vectors where the EELS signal is non-magnetic, thus only adding noise to the desired magnetic signal. Here, we modify the qE experimental set up by placing a double hole aperture, called DA in the diffraction plane and evaluate the EMCD signal obtained from this $\mathrm{qE}$ mode (Fig. 1c). In this way, we acquire EELS edge containing the magnetic signal reducing the sources of noise to their strict minimum. We compare the results from conventional $\mathrm{qE}$ mode with the ones from DA mode.

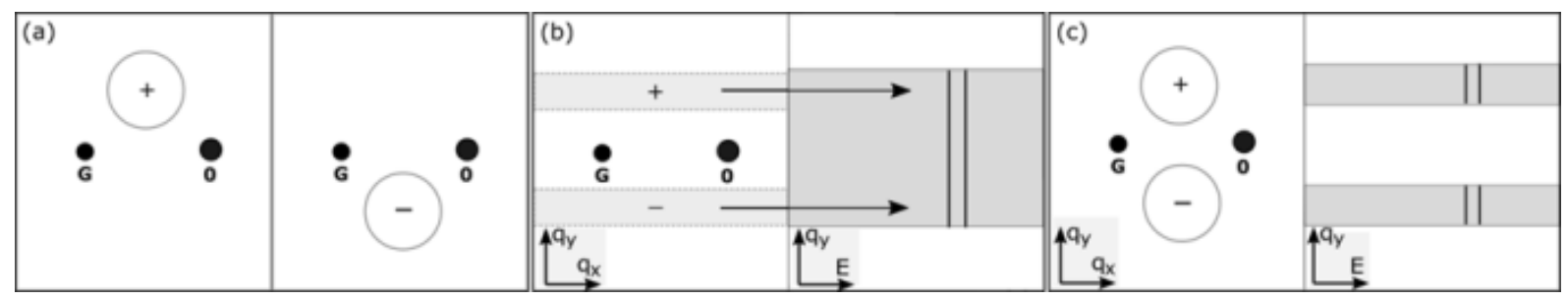

Figure 1: A schematic of geometrical conditions for (a) conventional EMCD (b) qE EMCD (c) DA EMCD 


\section{Experimental Details:}

We use a single crystalline bcc Fe sample to demonstrate the DA technique. For this purpose, a $25 \mathrm{~nm}$ thick Fe layer was epitaxially grown on a MgO (001) substrate using DC magnetron sputtering. The base pressure of the growth chamber was below $2 \times 10^{-9} \mathrm{mbar}$ and the operating pressure of the Ar gas (99.999 99\%) was $2.7 \times 10^{-3}$ mbar. Prior to the deposition, the MgO substrate was annealed at $550{ }^{\circ} \mathrm{C}$ for $1 \mathrm{~h}$. The substrate temperature during the growth was kept constant at $165^{\circ} \mathrm{C}$. The Fe film was capped with a $2.3 \mathrm{~nm}$ thick, $\mathrm{RF}$ sputtered $\mathrm{Al}_{2} \mathrm{O}_{3}$ layer to avoid oxidation. The sample was investigated in a Phillips X-Pert Pro MRD diffractometer $\left(\mathrm{Cu} \mathrm{K}_{\alpha}=1.5418 \AA\right.$ ). X-ray reflectivity (XRR) and diffraction (XRD) measurements are performed to investigate the thickness and interface roughness of the Fe layer as well as the mosaicity.

For EMCD experiments, the TEM sample was prepared in a plan view geometry by conventional sample preparation technique [15]. For this purpose, the sample was mechanically polished and dimple grinded from the substrate side to reduce the thickness $<20 \mu \mathrm{m}$. Finally Ar ion milling in a Gatan precision ion polishing system (PIPS) was used from the substrate side to further reduce the thickness. The energy of the ions was initially set to $4 \mathrm{keV}$ and then changed to $2 \mathrm{keV}$ when the perforation was near. With this procedure, we get a several micrometers wide sample area where the Fe thickness in the electron beam direction varies from a few nanometers at the edge of the hole to 25 $\mathrm{nm}$ at the interface where the $\mathrm{MgO}$ substrate starts.

The EMCD experiments were performed on a JEOL 2000FX containing an LaB6 filament attached with a Gatan energy filter GIF2002. The TEM sample had a thickness of $25 \mathrm{~nm}$ at the place of the EMCD analysis. The EMCD experiments were carried out in a 2 beam condition (2BC) with $g=(200)$ excited and with the DA inserted on the level of the entrance aperture of the GIF2002. The reasons for the choice of $\mathbf{G}= \pm(200)$ systematic row instead of $\mathbf{G}= \pm(110)$ is that in the (200) 2BC orientation the EMCD signal is stronger than in the (110) 2BC for a sample thickness between 20 to $30 \mathrm{~nm}$ [7]. To get maximum intensity in the diffraction plane, a convergent electron beam with a convergence semiangle $\alpha=2.5 \mathrm{mrad}$ was used to illuminate a $100 \mathrm{~nm}$ sample area. The sample was oriented such that the $\mathbf{G}=(200)$ is parallel to the qx axis in the diffraction plane for the reasons explained in the introduction part. Fig. 2a shows an energy filtered diffraction pattern (EFDIF) with the position of the beams used for DA acquisitions. This diffraction pattern was taken at an energy loss of $700 \mathrm{eV}$ with a $50 \mathrm{eV}$ energy slit. Fig. 2b shows the experimental image of the double hole aperture (DA). The collection semi-angle $\beta$ for each aperture was set to 4 mrad. For the qE-mode acquisitions, a different position of the beams was used by moving the direct beam to the edge of the CCD camera (Fig. 2d). The reason for choosing this position of the beams is that even in the 2BC, a weak magnetic signal exists between the 0 beam and $-\mathbf{G}$ which is of opposite nature to the magnetic signal between the 0 beam and the strongly excited $\mathbf{G}[5]$. This has been schematically illustrated in Fig. 2c. Thus acquiring the $\mathrm{qE}$ image with the beams position used for DA acquisitions (Fig. 2a) will result in an integration of both of these opposite magnetic signals, causing an overall reduction of the EMCD signal. 


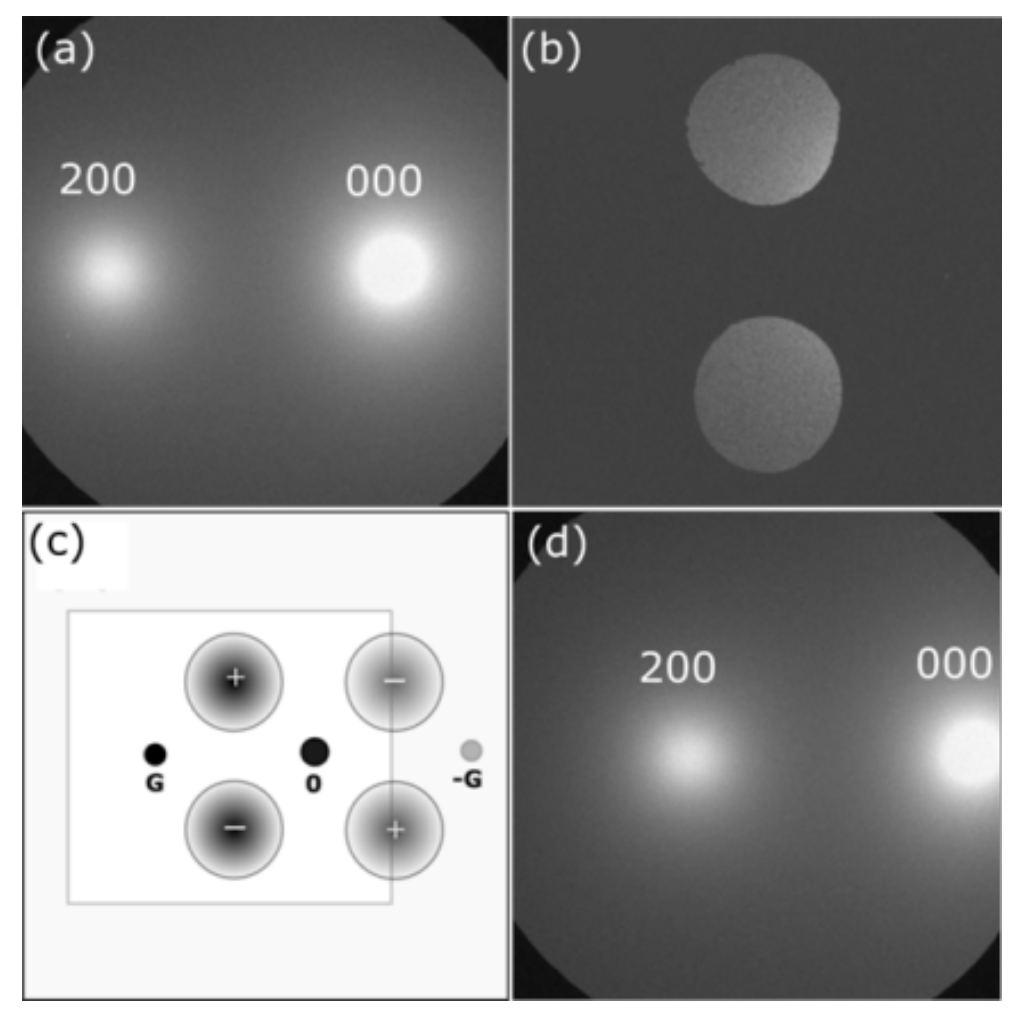

Figure 2: (a) The position of 2 beams for DA EMCD acquisitons (b) an experimental image of the DA (c) a schematic showing the presence of a weak magnetic signal across - $\mathbf{G}$ in 2BC with strongly excited $\mathbf{G}$ beam; (d) the position of 2 beams for qE EMCD acquisitions.

\section{Results:}

We compare the EMCD results obtained from DA and $\mathrm{qE}$ modes for bcc Fe sample. In the camera images (Fig. 3a \& 3b) of the EELS spectra acquired under DA and qE modes respectively, the tracks from the spectra are clearly observed. The Fe L3 and L2 edges can be seen in the form of bright lines in the images. The EELS spectra at the $\mathrm{C}+$ and $\mathrm{C}$-positions were extracted by integrating the line traces in the regions marked with blue and red boxes respectively. The intensities of the signals extracted from $\mathrm{C}+$ and $\mathrm{C}$ - spectra were both post edge normalized. Fig. 3c \& 3d show the normalized $\mathrm{C}+$ and $\mathrm{C}$ - spectra along with their difference signals (EMCD) for DA and qE modes respectively. A clear EMCD signal can be observed on the L3 and L2 edges for both of the acquisition modes. 

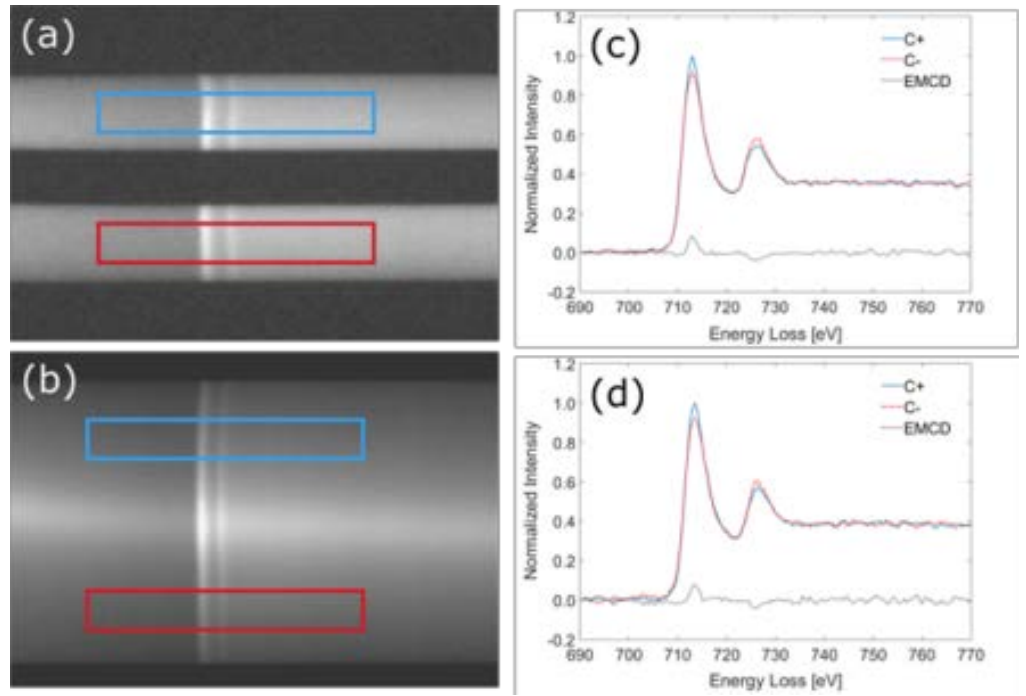

Figure 3: An experimental EELS image in (a) DA and (b) qE mode. EMCD spectra were obtained for (c) DA and (d) qE acquisition modes by extracting and post-processing the chiral (+,-) EELS spectra from the blue and red boxes respectively. The EELS images acquisition time was $10 \mathrm{~s}$. The EELS spectra were background subtracted by fitting a power law background model in the pre-edge region and extrapolating the background under the edges.

The EMCD signal strength in DA and qE modes is very close to each other with an L3 edge difference of $8.3 \%$ and $7.9 \%$ respectively. The qE EMCD signal appears to be noisier as compared to DA EMCD signal. To estimate the noise in the EMCD spectra, the standard deviation $\sigma$ in the pre edge region was calculated by fitting a window between $690 \mathrm{eV}$ and $705 \mathrm{eV}$. We obtain $\sigma=0.0046$ and $\sigma=0.0120$ for DA and qE EMCD spectra, respectively. It can be seen that the standard deviation of the noise is about a factor of 3 higher for the qE EMCD signal as compared to the DA EMCD signal. This effect of increased noise can also be seen directly in the EELS spectra in the form of increased background. Fig. 4 shows a comparison of intensity normalized EELS spectra extracted from $\mathrm{C}+$ positions of DA and qE images. For the same L3-peak height, the qE EELS has higher background signal as compared to DA EELS.

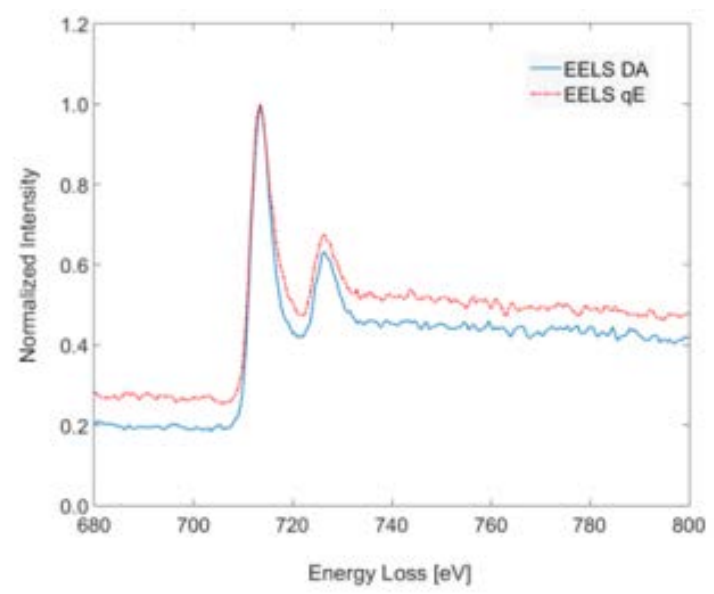

Figure 4: A comparision of EELS spectra extracted from C+ positions of DA and qE EELS images.

Apart from getting better signal to noise ratio $(\mathrm{S} / \mathrm{N})$ in $\mathrm{DA}$ EMCD as compared to $\mathrm{qE}$ EMCD for equal acquisition times, another advantage of DA acquisition mode is that we can use much longer acquisition times. In the $\mathrm{qE}$ acquisition mode, the intensity of the 0 and g beams in the EFDIFF pattern 
is integrated and appears in the middle of the L3 and L2 edges on the CCD camera (Fig. 3b). This intensity is much higher than the intensity at the chiral positions which is also apparent from the EFDIFF pattens shown in Fig. 2 and it limits the overall acquisition time for the qE EELS image. In our experimental set up, an acquisition time of $15 \mathrm{~s}$ was sufficient to saturate the CCD camera in the $\mathrm{qE}$ mode. Since in DA acquisition mode the intensities of 0 and g beams are cut out and only the signals at chiral positions are collected, it allows us to obtain acquisition times of more than $100 \mathrm{~s}$ for the same experimental set up. Fig. 5 shows an EMCD spectrum extracted from a DA image acquired with $60 \mathrm{~s}$ acquisition time. The EELS spectra extracted from the DA image were background subtracted and post edge normalized in the same way as described for the spectra of Fig. 3. The S/N for this EMCD spectrum is much higher than those acquired for $10 \mathrm{~s}$.

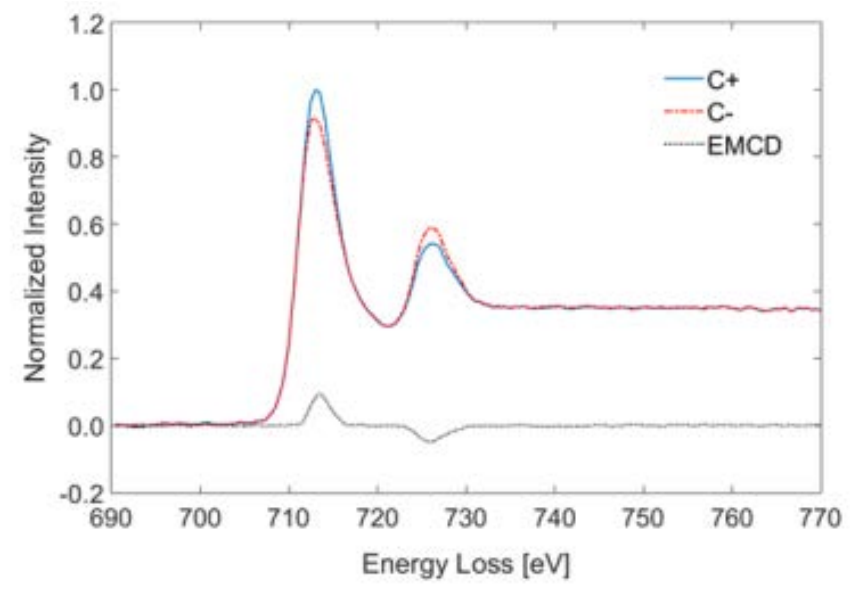

Figure 5: EELS spectra extracted from $\mathrm{C}^{+}$and $\mathrm{C}$ - positions of a DA EELS image with $60 \mathrm{~s}$ acquisition time along with their relative difference (EMCD) signal.

We applied sum rules to calculate the $m_{l} / m_{s}$ ratio for the EMCD spectrum shown in Fig. 5 . The $m_{l} / m_{s}$ value is calculated to be $0.06 \pm 0.01$. This value is in good agreement with the $\mathrm{m}_{\mathrm{l}} / \mathrm{m}_{\mathrm{s}}$ ratio measurements for bcc $\mathrm{Fe}$ obtained from XMCD 0.043 [16], gyromegnetic ratio measurements 0.044 [17], neutron scattering experiments 0.062 [18] and EMCD measurements [5][19][20][21]. Thus, using the DA acquisition geometry allows for low noise and simultaneous acquisition of the EELS spectra at the $\mathrm{C}+$ and $\mathrm{C}$ - positions enabling the measurement of the $\mathrm{m}_{\mathrm{l}} / \mathrm{m}_{\mathrm{s}}$ ratio.

\section{Discussions:}

In the classical EMCD technique, two chiral EELS spectra are acquired sequentially. If any experimental condition changes between the two acquisitions, it can be difficult to use the obtained information for reliable quantitative measurements. The DA acquisition mode allows for the acquisition of both the chiral EELS spectra at the same time under exactly the same physical and geometrical conditions. The $\mathrm{qE}$ acquisition mode also allows for the simultaneous acquisition of chiral EELS spectra, but it has a few disadvantages as compared to the DA acquisition mode. In the qE mode we integrate the real magnetic signal with the intensities from scatter vectors that do not contain a magnetic signal and thus degrade the S/N ratio of the resulting EMCD signal. Fig. 6a shows the beams position for $\mathrm{qE}$ acquisition mode as described in the experimental part. The white circles indicate the position of the DA. To estimate the additional signal which is integrated with the DA signal in the $\mathrm{qE}$ acquisition mode, we moved the DA to the positions shown by red and yellow circles. The corresponding chiral EELS spectra from the red and yellow circle positions are shown in Fig. 6b \& 6c. 
It can be seen that as we move away from the standard Thales circle positions to the red circle positions, the EMCD signal gets weaker with an increase in noise. As we move further away to the yellow circle positions, the EMCD signal disappears and the noise increases significantly.
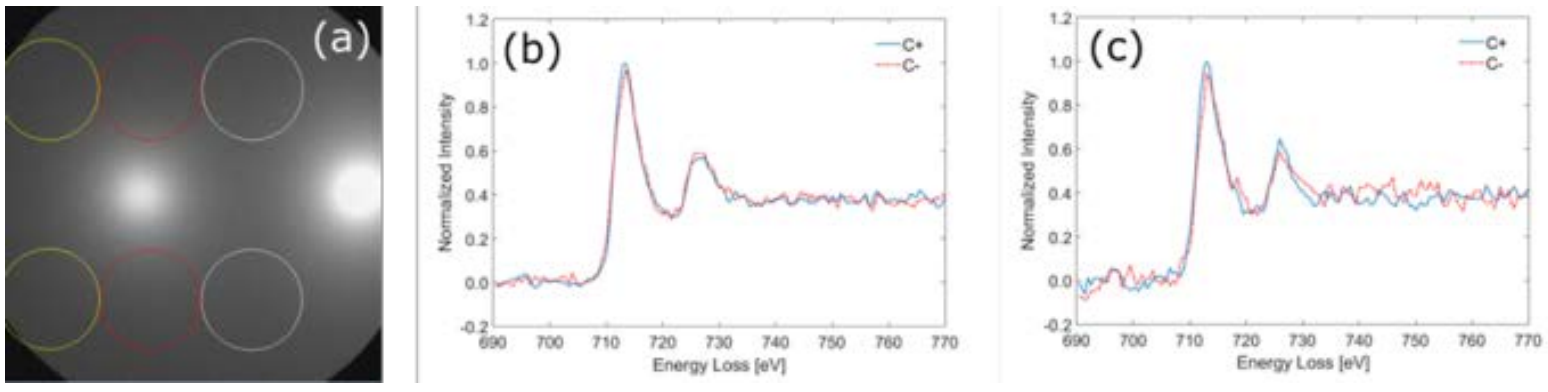

Figure 6: (a) Different positions of DA with respect to the position of 2 beams used for $\mathrm{qE}$ acquisitions. White circles show the positions used for DA acquisitions. (b) \& (c) chiral EELS spectra extracted from the EELS images obtained by shifting the DA to (b) red and (c) yellow circle's positions.

In addition to the additional noise in the $\mathrm{qE}$ mode as compared to DA mode, an aperture shift to a different position in reciprocal space might also lead to the fact that contributions from different magnetic signal arising from different sources are integrated [22].With the DA inserted, the detector size and position can be chosen in reciprocal space and thus spurious signals whose EMCD signal appears at different scatter vectors can be screened out.

\section{Conclusions:}

We have used a double hole aperture (DA) in the transmission electron microscope to acquire the simultaneous electron energy loss spectra (EELS) in the electron magnetic circular dichroism (EMCD) experiments. We calculated the $\mathrm{m}_{\mathrm{l}} / \mathrm{m}_{\mathrm{s}}$ ratio of $0.06 \pm 0.01$ for bcc Fe sample. The advantage of using the DA over the conventional EMCD geometry is that the EMCD signals can be acquired in a single acquisition under exactly the same experimental conditions. The DA experimental setup gives a clear improvement in S/N ratio of the EMCD signal as compared to the previously reported qE mode that also allows for the acquisition of two simultaneous EELS spectra. We show that implementing the DA allows us to use much longer acquisition times as compared to the $\mathrm{qE}$ acquisition mode which leads to an EMCD signal with a much higher S/N ratio. The DA equally filters out unwanted signals such as the high intensity 0 beam as well as spurious magnetic signals that can appear at other scatter angles and would be mixed with the wished magnetic signal in the qE mode. Hence using the DA, we can obtain an improved EMCD signal in terms of $\mathrm{S} / \mathrm{N}$ ratio as well as identical experimental conditions for the acquisition of both EMCD signal forming EELS spectra which is a step forward to improve quantitative EMCD measurements.

\section{Acknowledgements:}

We acknowledge the generous support of this work by the Swedish Science Council, contracts C0367901 and 2016-05259 as well as the Knut and Alice Wallenberg foundation. We thank the (c) Gatan, Inc. for helpful support in discussions and the spectrometer alignment. 


\section{References:}

[1] C. Hébert and P. Schattschneider, "A proposal for dichroic experiments in the electron microscope.,” Ultramicroscopy, vol. 96, no. 3-4, pp. 463-8, Sep. 2003.

[2] P. Schattschneider et al., "Detection of magnetic circular dichroism using a transmission electron microscope,” Nature, vol. 441, no. 7092, pp. 486-488, May 2006.

[3] J. Stohr et al., "Element-Specific Magnetic Microscopy with Circularly Polarized X-Rays," Source Sci. New Ser., vol. 259, no. 5095, pp. 658-661, 1993.

[4] T. Funk, A. Deb, S. J. George, H. Wang, and S. P. Cramer, "X-ray magnetic circular dichroism—a high energy probe of magnetic properties," Coord. Chem. Rev., vol. 249, pp. 330, 2005.

[5] H. Lidbaum et al., "Quantitative magnetic information from reciprocal space maps in transmission electron microscopy,” Phys. Rev. Lett., vol. 102, no. 3, pp. 1-4, 2009.

[6] P. Schattschneider, J. Verbeeck, and A. L. Hamon, "Real space maps of atomic transitions," Ultramicroscopy, vol. 109, no. 7, pp. 781-787, 2009.

[7] H. Lidbaum et al., "Reciprocal and real space maps for EMCD experiments," Ultramicroscopy, vol. 110, pp. 1380-1389, 2010.

[8] M. Stöger-Pollach, C. D. Treiber, G. P. Resch, D. A. Keays, and I. Ennen, "EMCD real space maps of Magnetospirillum magnetotacticum,” Micron, vol. 42, no. 5, pp. 456-460, 2011.

[9] T. Thersleff, J. Rusz, B. Hjörvarsson, and K. Leifer, "Detection of magnetic circular dichroism with subnanometer convergent electron beams," Phys. Rev. B, vol. 94, no. 13, p. 134430, Oct. 2016.

[10] T. Thersleff et al., "Towards sub-nanometer real-space observation of spin and orbital magnetism at the Fe/MgO interface,” Sci. Rep., vol. 7, p. 44802, 2017.

[11] J. Rusz et al., "Magnetic measurements with atomic-plane resolution,” Nat. Commun., vol. 7, no. May, p. 12672, 2016.

[12] Z. Wang et al., "Atomic scale imaging of magnetic circular dichroism by achromatic electron microscopy,” Nat. Mater., vol. 17, no. 3, pp. 221-225, Mar. 2018.

[13] P. Schattschneider, I. Ennen, S. Löffler, M. Stöger-Pollach, and J. Verbeeck, "Circular dichroism in the electron microscope: Progress and applications (invited),” J. Appl. Phys., vol. 107, no. 9, 2010.

[14] P. Schattschneider, C. Hébert, S. Rubino, M. Stöger-Pollach, J. Rusz, and P. Novák, "Magnetic circular dichroism in EELS: Towards $10 \mathrm{~nm}$ resolution," Ultramicroscopy, vol. 108, no. 5, pp. 433-438, 2008.

[15] R. Alani and P. R. Swann, "PRECISION ION POLISHING SYSTEM - A NEW INSTRUMENT FOR TEM SPECIMEN PREPARATION OF MATERIALS,” Mater. Res. Soc., vol. 254, pp. 43-63, Jan. 1992.

[16] C. T. Chen et al., "Experimental Confirmation of the X-Ray Magnetic Circular Dichroism Sum Rules for Iron and Cobalt,” vol. 75, 1995.

[17] D. Bonnenberg, K. A. Hempel, and H. P. J. Wijn, “1.2.1.2.4 Atomic magnetic moment, magnetic moment density, g and g' factor," in 3d, $4 d$ and $5 d$ Elements, Alloys and Compounds, Berlin/Heidelberg: Springer-Verlag, pp. 174-188. 
[18] M. B. Stearns, “1.1.2.7 Form factors, densities and magnetic moments," in $3 d$, $4 d$ and $5 d$ Elements, Alloys and Compounds, Berlin/Heidelberg: Springer-Verlag, pp. 52-57.

[19] J. Rusz et al., "Influence of plural scattering on the quantitative determination of spin and orbital moments in electron magnetic chiral dichroism measurements," Phys. Rev. B - Condens. Matter Mater. Phys., vol. 83, no. 13, pp. 1-4, 2011.

[20] L. Calmels et al., "Experimental application of sum rules for electron energy loss magnetic chiral dichroism,” Phys. Rev. B, vol. 76, no. 6, p. 060409, Aug. 2007.

[21] B. Warot-Fonrose, C. Gatel, L. Calmels, V. Serin, and P. Schattschneider, "Effect of spatial and energy distortions on energy-loss magnetic chiral dichroism measurements: Application to an iron thin film,” Ultramicroscopy, vol. 110, no. 8, pp. 1033-1037, 2010.

[22] T. Thersleff et al., "Quantitative analysis of magnetic spin and orbital moments from an oxidized iron (1 10 ) surface using electron magnetic circular dichroism," Sci. Rep., vol. 5, no. 1, p. 13012, 2015. 